\title{
PERTURBATION OF LINEAR OPERATORS BY IDEMPOTENTS
}

\author{
Mícheál Ó Searcóid
}

It is a curious fact that, given any square matrix with entries from a given field, it is possible to produce an invertible matrix simply by subtracting 1 from some of the diagonal entries of the matrix. (There is of course nothing special about 1 here; any non-zero member of the field could be used.) An inductive proof is given in Proposition 1 below. The proof is effected by playing off the two idempotents of the field against one another.

Proposition 1. Suppose $n$ is a natural number and $A$ is an $n \times n$ matrix. Then there exists a diagonal idempotent $n \times n$ matrix $Q$ such that $A-Q$ is invertible.

Proof: The result is trivial if $n=1$. Suppose it is true for $n=$ $m \geq 1$ and let $\left(\begin{array}{cc}\alpha & f \\ z & V+P\end{array}\right)$ represent an arbitrary $(m+1) \times(m+1)$ matrix, where $\alpha$ is a scalar, $f$ is a $1 \times m$ matrix, $z$ is an $m \times 1$ matrix, $V$ is an $m \times m$ invertible matrix and $P$ is an $m \times m$ diagonal idempotent matrix. Note that

$$
\left(\begin{array}{cc}
\alpha & f \\
z & V+P
\end{array}\right)=\left(\begin{array}{cc}
\alpha & f \\
z & V
\end{array}\right)+\left(\begin{array}{cc}
0 & 0 \\
0 & P
\end{array}\right)=\left(\begin{array}{cc}
\alpha-1 & f \\
z & V
\end{array}\right)+\left(\begin{array}{cc}
1 & 0 \\
0 & P
\end{array}\right) .
$$

Both $\left(\begin{array}{cc}0 & 0 \\ 0 & P\end{array}\right)$ and $\left(\begin{array}{cc}1 & 0 \\ 0 & P\end{array}\right)$ are diagonal idempotent matrices; moreover since the determinants of the matrices $\left(\begin{array}{cc}\alpha-1 & f \\ z & V\end{array}\right)$ and $\left(\begin{array}{cc}\alpha & f \\ z & V\end{array}\right)$ differ by the determinant of $V$, which is non-zero, one or other of the matrices is invertible. It follows that the result is true for $n=m+1$, and the general result follows by induction. 
Let us turn now to infinite dimensions, where we shall use the notation $\mathcal{L}(X, W)$ to denote the set of linear operators from any linear space $X$ to any linear space $W$ and abbreviate this to $\mathcal{L}(X)$ if $X=W$. The following result is well known.

Proposition 2. Suppose $Y$ and $X$ are linear spaces over the same field and $T \in \mathcal{L}(Y \oplus X)$ is represented matricially by $T=$ $\left(\begin{array}{ll}A & B \\ C & V\end{array}\right)$, where $A \in \mathcal{L}(Y), B \in \mathcal{L}(X, Y), C \in \mathcal{L}(Y, X)$ and $V \in \mathcal{L}(X)$. Suppose $V$ is invertible in $\mathcal{L}(X)$; then $T$ is invertible if and only if $A-B V^{-1} C$ is invertible in $\mathcal{L}(Y)$.

Proof: It is easy to check that if $A-B V^{-1} C$ is not invertible then $T$ is not invertible. If $A-B V^{-1} C$ is invertible, we set $J \in \mathcal{L}(Y)$ to be its inverse; then a short calculation will confirm that

$$
\left(\begin{array}{cc}
J & -J B V^{-1} \\
-V^{-1} C J & V^{-1}+V^{-1} C J B V^{-1}
\end{array}\right)
$$

is inverse to $T$.

When $Y$ is finite dimensional, the condition of Proposition 2 is, of course, equivalent to $\operatorname{det}\left(A-B V^{-1} C\right) \neq 0$, which reduces to $A \neq B V^{-1} C$ when $Y$ is one dimensional. This can be used instead of the determinant argument in Proposition 1 and might lead us to believe that a statement analogous to Proposition 1 is true for operators on infinite dimensional spaces. Specifically, suppose $X$ is a linear space, $\mathcal{E}=\left\{e_{i}: i \in I\right\}$ is any basis for $X$ and $T \in \mathcal{L}(X)$; is it in general possible to find an idempotent $Q \in \mathcal{L}(X)$ with $Q e_{i} \in\left\{e_{i}, 0\right\}$ for each $i \in I$ such that $T-Q$ is invertible? The answer is no, and the reader can easily verify the impossibility when $I$ is the set of natural numbers and $T$ is the unilateral shift, i.e., when $T$ satisfies the equations $T e_{i}=e_{i+1}$ for each $i \in \mathbf{N}$.

What happens when we drop the diagonal requirement? It was upon hearing from Tom Laffey that it has recently been proved that every operator on a space of countable dimension can be perturbed by an idempotent to produce an invertible operator that my interest in the question was aroused. The condition of countability is unnecessary; in Proposition 3 below we give a proof that 
the perturbation is possible for every operator on any linear space. It might be of interest to note that the proof of Proposition 3 is motivated by that of Proposition 1; induction has been replaced by Zorn's lemma and we again use the trick of playing off the zero against the identity, though not with the same degree of ostentation.

Proposition 3. Let $X$ be a linear space over a field F. Suppose that $T \in \mathcal{L}(X)$, the algebra of linear operators on $X$. Then there exists $P \in \mathcal{L}(X)$, with $P=P^{2}$, such that $T-P$ is invertible in $\mathcal{L}(X)$.

Proof: Let $\mathcal{S}$ denote the set of all ordered pairs $(M, Q)$ where $M$ is a subspace of $X$ invariant under $T, Q^{2}=Q \in \mathcal{L}(M)$, $(T-Q) M=M$ and $T_{M}-Q$ is injective, where $T_{M}$ denotes the restriction of $T$ to $M$. Note that $\mathcal{S} \neq \emptyset$ since $(\{0\}, 0) \in \mathcal{S}$.

Define a partial ordering on $\mathcal{S}$ by setting $\left(M_{i}, Q_{i}\right) \leq\left(M_{j}, Q_{j}\right)$ whenever both are in $\mathcal{S}, M_{i} \subseteq M_{j}$ and $Q_{i}$ is the restriction of $Q_{j}$ to $M_{i}$.

Suppose $\left\{\left(M_{i}, Q_{i}\right): i \in I\right\}$ is a totally ordered subset of $\mathcal{S}$. Then $Q$ is well-defined in $\mathcal{L}\left(\bigcup M_{i}\right)$ by setting $Q x=Q_{i} x \quad(i \in$ $\left.I, x \in M_{i}\right)$, and it is easy to check that $\left(\bigcup M_{i}, Q\right) \in \mathcal{S}$ and that $\left(M_{i}, Q_{i}\right) \leq\left(\bigcup M_{i}, Q\right)$ for all $i \in I$. It follows from Zorn's Lemma that there exists a maximal element $(Y, P)$ in $\mathcal{S}$. We claim that $Y=X$.

Firstly, suppose there were to exist $x \in X \backslash Y$ such that $T x \in$ $Y$. Then we could set $P x=x$ and extend $P$ linearly to $Y \oplus \mathbf{F} x$. It is an easy matter to check that we should then have $(Y \oplus \mathbf{F} x, P) \in$ $\mathcal{S}$, contradicting maximality of $(Y, P)$.

Secondly suppose there were to exist a polynomial $p$ with nonzero constant term and a vector $x \in X \backslash Y$ such that $p(T) x \in Y$; then we might assume $p$ and $x$ to satisfy these criteria with the degree $m$ of $p$ being the least possible for any such arrangement. It would follow that $\left\{T^{k} x: 0 \leq k<m\right\}$ was a linearly independent set and that the subspace $W$ of $X$ spanned by it satisfied $W \cap Y=$ $\{0\}$. We could set $P=0$ on $W$ and extend $P$ linearly to $W \oplus Y$. Using the fact that the constant term of $p$ was specified to be nonzero, it is easy to check that we should then have $(Y \oplus W, P) \in \mathcal{S}$, 
again contradicting maximality of $(Y, P)$.

Thirdly suppose there were to exist $x \in X \backslash Y$ such that $p(T)(x) \in X \backslash Y$ for every non-zero polynomial $p$. Then certainly $\left\{x, T x, T^{2} x, \ldots\right\}$ would form a linearly independent set. Letting $V$ denote the subspace of $X$ spanned by these vectors, we could define $Q$ to be the linear operator on $V$ for which $Q T^{2 n} x=T^{2 n} x$ and $Q T^{2 n+1} x=T^{2 n+2} x-T^{2 n} x,(n \geq 0)$. Then we should have $Q^{2}=Q$ and it is easy to check that $T_{V}+I_{V}-Q$ would be inverse to $T_{V}-Q$ in $\mathcal{L}(V)$, where $I_{V}$ would denote the identity operator on $V$ and $T_{V}$ the restriction of $T$ to $V$. It would follow that $(Y \oplus V, P \oplus Q) \in \mathcal{S}$, yet again contradicting the maximality of $(Y, P)$.

We must conclude that $Y=X$. Then $T-P$ is bijective and therefore invertible in $\mathcal{L}(X)$.

In conclusion, let me add one observation which might be of interest. This is that, in many cases, we must look for a perturbing idempotent with infinite range. Indeed, if the index ind $(T)$ of an operator $T$ is defined to be the difference between the dimension of its kernel and the co-dimension of its range whenever these quantities are finite, it can be shown that ind $(T+F)=\operatorname{ind}(T)$ for every operator of finite rank $F$. (The reader who is not familiar with this result might like to while away a little time in providing a proof.) Since every invertible operator has zero index and the unilateral shift has index -1 , it is easy to check that the observation is correct.

Mícheál Ó Searcóid

Department of Mathematics

University College

Dublin

email: micheal.osearcoid@ucd.ie 